\title{
Arbitrary Signals and Cognitive Complexity
}

\author{
Ronald J. Planer and David Kalkman
}

\begin{abstract}
The arbitrariness of a signal has long been seen as a theoretically important but difficult to pin down notion. In this article, we suggest there are at least two different notions of arbitrariness at play in philosophical and scientific debates concerning the use of arbitrary signals, and work towards improved analyses of both. We then consider how these different types of arbitrariness can co-occur and come apart. Finally, we examine the connections between these two types of arbitrariness and the cognitive complexity of signal users with an eye towards better evaluating one possible form of humannonhuman communicative continuity. We show that each type of arbitrariness bears its own nuanced relationship to cognitive complexity, demonstrating the theoretical importance of keeping these two notions separate.
\end{abstract}

\section{Introduction}

\section{Arbitrariness $_{A}$}

3 Arbitrariness $_{R}$

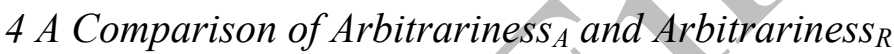

5 Arbitrariness $_{A}$, Arbitrariness, and Cognitive Complexity

\subsection{Arbitrariness A $_{\text {and }}$ cognitive complexity}

\subsection{Arbitrariness $_{\mathrm{R}}$ and cognitive complexity}

\section{Introduction}

At least in philosophy of science circles, there has been an appreciation for some time that the notion of arbitrariness is, as Godfrey-Smith ([2000], p. 205) has put it, 'a useful if elusive concept'. Godfrey-Smith was commenting on the use of this notion in genetics and cell biology, but in our view, the conceptual issues that arise in connection with arbitrariness in that domain extend to others. In this article, we attempt to make progress on the task of analysing the notion of arbitrariness as it relates to signalling and communication at all levels of the biological hierarchy. Before getting started, 
however, a brief discussion of the theoretical role of arbitrariness - of the work the notion does or might do-is in order.

In philosophy of biology, arbitrariness has been seen as intimately connected to a cluster of issues involving informational description and explanation. Perhaps the most common idea has been that a signal's being arbitrary justifies according semantic information to it, as opposed to mere correlational information. ${ }^{1}$ This role for arbitrariness is most clearly evident in debates concerning the informational status of genes. ${ }^{2,3}$ Stegmann ([2004], p. 206) nicely summarizes this line of thinking.) As he explains, the idea appears to be:

[...] based on an analogy between chemical and linguistic arbitrariness. Linguistic arbitrariness expresses the fact that the linguistic properties of a word are usually not naturally related to its meaning. The phonetic form of 'dog' does not reflect a property of dogs. In Peircean terms, the relation between a word and its meaning is symbolic. Similarly, the genetic code's arbitrariness is understood as the absence of a natural connection between codons and amino acids. Chemical arbitrariness arguably establishes a language-like symbolic relation between codons and amino acids. It is then thought legitimate to attribute meaning and semantic information to genes or its components.

A related, but considerably stronger idea is that arbitrariness is a necessary condition for a physical structure's being a signal at all. ${ }^{4}$ The details are seldom worked out, but the idea seems to be something like this: (i) a structure counts as a signal only if an informational explanation of the functioning of that structure in some interaction

${ }^{1}$ Put roughly, one thing carries correlational information about another when the former raises the probability of the latter.

${ }^{2}$ To our knowledge, Maynard Smith ([2000]) was the first to defend this idea as it applies to genes.

${ }^{3}$ For a general application of this idea, see (Cao [2012]). Artiga ([2016]) also expresses some sympathy for using this idea to pick out mental representations.

${ }^{4}$ We read Scarantino ([2013]) as in effect defending such a proposal. Godfrey-Smith ([2014]) can also be interpreted in this way. 
contributes something additional to our understanding than is contributed by a purely physical explanation, and (ii) an informational explanation does this only in cases where the structure is arbitrary. One might alternatively think that there is a tight connection between arbitrariness and informational explanation, but reject the idea that an informational explanation must yield something over and above a physical explanation in order for the relevant structure to count as a signal. ${ }^{5}$ Then arbitrariness would be playing a simpler theoretical role: its presence or absence would explain why it is that we sometimes find informational explanations so useful.

Arbitrariness has been much discussed in other literatures too, though these discussions have tended to focus on a different set of foundational issues. In particular, theorists concerned with the origins of human language have often been interested in the issue of arbitrariness as it applies to animal signals. Those who think human language is continuous with animal signalling often draw attention to certain animal signals that are plausibly regarded as arbitrary. The thought here is that since human language words are in general arbitrarily related to their contents, the existence of arbitrary animal signals would imply some form of continuity with human language. ${ }^{6}$ Sceptics then respond to this by attempting to show that the signals in question are not so arbitrary after all.

A good example of this back and forth is visible in debates surrounding the putatively semantic or symbolic status of vervet monkey alarm. The following passage by Price et al. ([2015], pp. 1-2) nicely expresses the initial excitement among animal psychologists surrounding the discovery of the vervet alarm call system:

\footnotetext{
${ }^{5}$ We think Skyrms is perhaps an example of this, though he has also been interpreted as holding the preceding view (see, for example, Godfrey-Smith [2013a]; Sterelny [2012]).

${ }^{6}$ See, for example, Seyfarth et al.'s ([1980]) seminal article.
} 
Because of their [that is, vervet alarm calls] potential relevance for understanding the origins of human speech, the findings were interpreted within a linguistic framework. According to semiotic theory, the relationship between a signifier and the signified can take three modes. They can be classified as indexical, when the signifier is in some way physically or causally linked to the signified, like smoke is linked to fire. In the iconic mode, the signifier bears a physical resemblance to the signified, whereas in the symbolic mode, the relationship between signifier and the signified is arbitrary and purely conventional. Because the vervet monkey, alarm calls bore no resemblance to their respective predator category, in the sense that they did not mimic the sounds made by the respective predators, they were deemed to be non-iconic and thus arbitrary. As arbitrariness constitutes one of the key criteria for symbolic communication, the monkey alarm calls were seen as the first example of symbolic communication in nonhuman animals

This line of thought has been influential with certain philosophers as well, though the main ideas have been spun in a somewhat different way. Specifically, a connection has been made between animal communication systems like the vervets' and Lewis's ([2002]) seminal work on convention, Take, for example, the following discussion by Skyrms ([1998], p. 411), the leading voice from within this camp, who begins:

The first step in the formation of language is the first step in the formation of a convention. Predator-specific alarm calls can serve a useful purpose only if the animal giving a call and the animal receiving the call associate types of predators with types of calls in the same way. There are always many alternative patterns of association that would serve to transmit the information. How is a particular pattern initially selected?

In line with Price et al., Skyrms ([1998], p. 411) then notes that alarm calls such as the vervets', 'do not seem to be imitations of the calls of the associated predator', and likely never were. This leads him to claim that such alarm call systems are purely conventional. Skyrms then shows how the emergence of such conventions among animals can be explained using Lewis-style signalling games. The simplest games of 
this sort feature just two equiprobable states of the world $\left(w_{1}, w_{2}\right)$ two signals $\left(s_{1}, s_{2}\right)$, and two acts $\left(a_{1}, a_{2}\right)$. The sender observes the state and produces one of her two signals, while the receiver observes the signal and produces one of his two acts. There is a single act that is right for each state and both sender and receiver agree on which act that is. Here there are two equally good signalling systems (taking numerical agreement among state and act to indicate a correct match):

(1) $w_{1} \rightarrow s_{1} \rightarrow a_{1} ; w_{2} \rightarrow s_{2} \rightarrow a_{2}$,

(2) $w_{1} \rightarrow s_{2} \rightarrow a_{1} ; w_{2} \rightarrow s_{1} \rightarrow a_{2}$.

Hence each has an equally good alternative. The thought, then, is that the selection of any one of them is arbitrary, and so conventional. This is true whether it is rational choice on the part of the agents that leads them to the convention, or some simpler selective force such as basic reinforcement learning.

Others have looked at this attitude regarding animal alarm calls and found it to be quite wrongheaded. For example, in a now quite influential critique, Owren et al. ([2010], p. 770) write:

[...] a typical approach has been to argue that the acoustics of vocalizations such as vervet alarm calls bear little or no discernable relationship to their respective referents. Yet vocalizations such as predator alarms appear to be anything but arbitrary. As is the case with frogs, the acoustics of these calls are well-designed for detection and localizations, and likely also prime listeners by engaging low-level attention and arousal mechanisms. Regardless of whether the calls are used narrowly or broadly, alarm vocalizations in many primates are short with abrupt onsets, noisy or with strong pitch modulation. These widely shared acoustic features are ideally suited for capturing and manipulating listener attention and arousal via short, direct links from the auditory 
periphery to brainstem regions regulating whole-body activation. As a result, alarm-call acoustics alone tend to elicit orienting responses and preparatory movements $[\ldots]$

We think a moment's reflection shows that there cannot be a single notion of arbitrariness in play across all of these contexts. The easiest way to see this is to simply note that there are many paradigm cases of signalling where the relevant physical structures are not arbitrary at all; if one person pantomimes the act of steering in order to request a ride home from her friend (and this is understood), surely she has produced a signal, that signal has semantic content, and an informational explanation of the interaction is illuminating. Less obvious, we think, is that different notions of arbitrariness are sometimes at play within the context of a single debate; we think this is true of the debate concerning the arbitrariness of vervet alarm calls, for example.

More specifically, we think there are at least two notions of arbitrariness- two different ways of lacking a 'natural connection' to a content - that are often run together, probably in part because human language words are paradigm examples of both. One concerns the existence of alternative structures that might have been used in the place of an actual signal. ${ }^{7}$ As will become clear below, it is far from trivial to turn this notion of arbitrariness into a precise concept. We will outline some of the conceptual difficulties we see before offering a working account of our own.

The other notion of arbitrariness concerns a lack of resemblance between a signal and its content. More precisely, the idea is that a signal is arbitrary in this sense just in

\footnotetext{
${ }^{7}$ We shall use the phrase 'in the place of the actual signal' repeatedly in what follows. One might take issue with this phrase on the grounds that it effectively rules out using arbitrariness to establish the signalhood of some actual structure under consideration. One can easily regain this conceptual possibility, however, by replacing the above language with the phrase 'in the place of the actual physical structure whose status as a signal is currently under consideration' (and perhaps a few other minor tweaks, depending on the exact context). We work with the former simply for economy's sake. (Similar remarks apply to the more formal characterization we give to this notion of arbitrariness below.)
} 
case it is non-iconic. Given that this notion is characterized by a lack of iconicity, a precise version of this notion requires clarity about the nature of iconicity. Here too we think there are some unresolved conceptual issues, and after outlining them, we offer an account that is sensitive to them.

The main objective of this article, then, is to thoroughly separate out these two notions and offer improved analyses of both. There are many philosophical and scientific questions that one might then use these analyses to shed light on-we have seen a few already. Here we will limit our focus to the goal of articulating the connections between arbitrariness and the cognitive complexity of signal users. This will enable us to better evaluate at least one form of potential human-nonhuman communicative continuity in this area involving arbitrariness. It is also one of the more tractable questions that might be taken up, and hence a sensible question to pursue in addition to the main work of this article. As will become clear, each form of arbitrariness bears its own, nuanced relationship to cognitive complexity. This serves as a kind of minimal demonstration of the importance of distinguishing the two forms of arbitrariness. We plan take up the more ambitious questions regarding, for example, informational explanation, in future work.

\section{Arbitrariness $_{A}$}

As was just mentioned, we think one notion of arbitrariness concerns the existence of alternative structures that might have been used in the place of some actual signal. So, for example, the English word 'dog' is arbitrary in the sense that some other type of acoustical pattern—'cat', say—might have been used to mean DOG.

Now obviously, this notion can be expressed in an all-or-nothing way - a signal is arbitrary just in case there exists at least one alternative-but often we are also 
concerned with the number of alternatives. Thus, we might say that the arbitrariness of a signal is proportional to the number of alternative structures that might have been used in its place; the larger this number of alternatives, the more arbitrary the signal; the smaller, the less arbitrary. So, 'dog' is a highly arbitrary signal in the sense that there is a huge number of other acoustical patterns that English speakers might have used to mean DOG.

We shall refer to this notion of arbitrariness in both its absolute and graded forms as 'alternative arbitrariness' and denote it as 'arbitrariness ${ }_{\mathrm{A}},{ }^{8}$ (Unless otherwise noted, it will be the graded version we have in mind when we speak of arbitrariness $\mathrm{A}_{\mathrm{A}}$.) At first blush, arbitrariness ${ }_{\mathrm{A}}$ appears to be a rather straightforward notion. But closer inspection reveals some thorny conceptual issues. In particular, it is not clear how to evaluate claims of the form, 'such-and-such an alternative might have been used in the place of the actual signal'. Might have been relative to what?

Below we will propose an answer. But before going there, we want to pause to emphasize that this question - the question of how to evaluate the various possibility claims on which the arbitrariness ${ }_{\mathrm{A}}$ of signal turns - is seldom recognized, much less wrestled with by theorists in this area. This is a real issue with the literature, in our view, as the notion of arbitrariness $_{\mathrm{A}}$ would seem to be of dubious theoretical value at best unless this question can be answered in a principled way. This point stands independently of any of our positive remarks.

Let us begin with a simple answer whose shortcomings are illuminating. One natural thought is that possibility claims of the above sort should be interpreted simply as expressing biological possibility. Thus, the idea here would be that a claim of the

\footnotetext{
${ }^{8}$ And we use 'arbitrary ${ }_{\mathrm{A}}$ ' for 'alternative arbitrary'.
} 
form, 'such-and-such an alternative might have been used in the place of the actual signal', is true just in case there exists a biologically possible world in which this alternative is used in the place of the actual signal.

We see two problems with this answer. First, it is unclear what the notion of biological possibility actually comes to. This notion is typically explicated by analogy with physical and chemical possibility; just as physically and chemically possible worlds are those worlds that are compatible with the laws of physics and chemistry, respectively, biologically possible worlds are supposed to be those worlds that are compatible with the laws of biology. The problem is, as many philosophers of biology have pointed out, ${ }^{9}$ there do not seem to be biological laws in the standard sense of the term 'law'. Biology does feature various regularities, but they are seldom exceptionless, and in general, they are contingent upon evolutionary history itself. Thus, it is unclear whether the notion of biological possibility is even a coherent one.

Second, and much more significantly in our view, even if we had some unproblematic way of spelling out biological possibility, we think it would be far too weak to capture the sorts of facts that are of interest to theorists in this area. The main reason is this: on any reasonable explication of biological possibility, there will in general be nothing biologically impossible about the relevant senders and receivers of a signal having very different physiologies, perceptumotor capacities, and cognitive capacities, nor anything biologically impossible about them inhabiting a very different set of environmental conditions. The problem is that when theorists judge some signal to be $\operatorname{arbitrary}_{\mathrm{A}}$ to such-and-such an extent, they tend to hold fixed most of the features of the actual communicative context (see, for example, the passage by Owren et al.

\footnotetext{
${ }^{9}$ See (Godfrey-Smith [2013b]) for a nice overview.
} 
above). The fact that senders or receivers with very different properties and/or inhabiting a very different environment might have used some other structure to communicate a focal message is not at all surprising. (We think the same problems attach to the proposal that the relevant possibility claims should be interpreted as expressing psychological possibility. Such a proposal would have the added draw-back of applying only to cases where signalling is meditated via psychological states.)

What this second problem shows is that the relevant notion of possibility must be much more fine-grained than mere biological possibility; the particulars of the communicative context under consideration must somehow play a stronger role. This suggests something like the following answer to our question: a claim of the form 'such-and-such an alternative might have been used in the place of the actual signal' is true just in case, given conditions relevantly similar to the actual ones, the alternative might have become the signal for the content that is communicated by the actual signal.

An informative version of this answer requires a specification of the ways that the two sets of conditions must be similar, and how similar they must be. We take the following account of arbitrariness A $_{\mathrm{A}}$ to be a plausible way of filling in these blanks, though we do not claim that this is the only plausible way of way of doing so.

Arbitrariness $_{\mathrm{A}}$ : The arbitrariness of structure $S$ as a signal for content $C$ is proportional to the number of alternative structures such that for each alternative $S_{i}$ in this range: (i) if, at the start of the process of signal creation, senders had instead been disposed to produce $S_{i}$ under the same conditions that they were disposed to 
produce $S$, then $S_{i}$ would have become the signal for $C$; and (ii) the antecedent of

(i) describes a biologically realistic situation. ${ }^{10}$

Let us consider both clauses, respectively. The antecedent of (i) describes a counterfactual situation in which, at the start of the process of signal creation, rather than being disposed to produce $S$ in response to some set of internal or external conditions, senders were instead disposed to produce $S_{i \cdot}{ }^{11}$ The conditional then says that were such a situation to have obtained, $S_{i}$ would have gone on to become the signal for $C$ in the relevant population. ${ }^{12}$

Why do we say it is the start of the process of signal creation that is relevant here? And what exactly is meant by this? Taking these in reverse order: Our account of arbitrariness $_{\mathrm{A}}$ is intended to cover signalling at both the population and individual level. In both cases, we can think of the 'start' of the process of signal creation as the initial appearance of the behaviour (construed in a broad way so as to include not just acts in

${ }^{10}$ The arbitrariness ${ }_{\mathrm{A}}$ of a signal will depend on one's scheme for individuating signals. (We briefly return to this point at the end of Section 4.) The only constraint imposed by our definition is that this scheme be a physical one (in a broad sense of 'physical') rather than a semantic one. There are obviously many ways of carving up physical structure space, and we suspect that different cases or contexts will call for different individuation schemes; a scheme that makes good sense for one communication channel, for example, might make less sense for another. Hence, we intend to remain flexible on this issue. The arbitrariness $_{\mathrm{A}}$ of a signal can thus be understood as being implicitly relativized to an individuation scheme for physical structure types. Having drawn attention to this issue, however, we propose to largely set it aside in what follows.

${ }^{11}$ If there are different $S$-involving dispositions across individuals, then matters are somewhat more complicated, though not intractably so.

${ }^{12}$ Here we are drawing inspiration from an important passage in (Lewis [2002], p. 70):

[...] there is no such thing as the only possible convention. If $\mathrm{R}$ is our actual convention, $\mathrm{R}$ must have the alterative R', and R' must be such that it could have been our convention instead of $\mathrm{R}$, if only people had started off conforming to $\mathrm{R}^{\prime}$ and expecting each other to [...] Any convention is arbitrary because there is an alternative regularity that could have been our convention instead.

To a good approximation, one can view our account of arbitrariness ${ }_{\mathrm{A}}$ as a kind of 'evolutionary' and 'reinforcement learning-friendly' development of Lewis's idea (though we are leaving aside the issue of conventions). 
the normal sense but also enduring phenotypic traits) that went on to become the signal, where there is a causal chain linking that event and the current use of the signal. ${ }^{13}$ (This event might be salient only in retrospect.) However, the process of signal creation can be quite different depending on which level we are dealing with. At the population level, this process might be a protracted one, with the behaviour not counting as a signal for such-and-such a content until it has been adopted by a sufficient number of individuals. In the case of animal signalling, for example, this might require natural selection to act on either the sender or receiver side, transforming a behaviour that was initially a cue or coercive act into a signal. ${ }^{14}$ At the individual level, signals might arise either via reinforcement learning on the part of sender and/or receiver, as in cases of ontogenetic ritualization, or directly, as when two humans lacking a shared communicative convention of the appropriate sort improvise a signal. In the latter case, talk of 'the start of the signal creation' is a bit awkward, but not in a problematic way we think. The process is exhausted by the production and interpretation of the signal by the sender and receiver, respectively, with the 'start' being the behaviour produced by the sender.

As for why it is the start of the process that is relevant, we think this for two main reasons: First, receivers might undergo various evolutionary or developmental changes that render them inflexible in the face of alternative structures at some later point in time; in particular, hardware or behavioural routines specialized for detecting and responding in a certain way to $S$ might evolve or develop. In this way, a structure that

\footnotetext{
${ }^{13}$ We include the causal chain requirement to rule out cases where the behaviour arises, but is then lost, only to be 'rediscovered' at a later time.

${ }^{14}$ Selection on one or both sides may be integral to the signal's coming to have the information content it does; senders may evolve to produce the behaviour in certain ways only because receivers have evolved to respond to that behaviour in certain ways, and so on.
} 
was a genuine alternative at an earlier time may cease to be so at a later one. We do not wish to rule such structures out. And going the other way, we also think that some structure's being a genuine alternative only at some later time should not count towards the $\operatorname{arbitrariness}_{\mathrm{A}}$ of the actual signal. Imagine, for example, that members of the population become more behaviourally flexible as time goes on. And imagine that this additional flexibility makes possible the use of some alternative structure in the place of the actual one. What matters, in our view, is whether the actual signal was forced on them at the start of the process of signal creation; if so, then even if some alternative to it becomes feasible at some later point in time, there is still a clear sense in which the actual signal is not arbitrary. ${ }^{15}$

A key feature of this definition is that it holds fixed virtually all of the facts about the actual communicative context as that context existed at the start of the process of signal creation. Structures that, for whatever reason, would not have 'gotten off the ground' as signals do not count as genuine alternatives to the actual signal, and hence do not contribute to the arbitrariness ${ }_{A}$ of the signal. But our definition adds a further constraint, expressed by clause (ii). This clause says that the counterfactual situation described by the antecedent of (i) is a biologically realistic situation. The intuitive idea here is this: in imagining that at the start of the process of signal creation, senders were instead disposed to produce $S_{i}$ under the same conditions as they were disposed to produce $S$, we are remaining within reasonable bounds, given the actual properties of

15 One might object at this point that our analysis of arbitrariness ${ }_{A}$ seems somewhat stipulative. Specifically, one might want the arbitrariness $_{\mathrm{A}}$ of a signal to also reflect the range of alternatives that could, given the current circumstances, go on to replace the actual signal. We are inclined to see these as two different versions of the same concept; a backward- and forward-looking version of arbitrariness $_{\mathrm{A}}$. In this article, we shall limit our focus just to the backward-looking version, as this seems to be the more common notion. But we take our analysis of the backward-looking notion to provide insight into how the forward-looking notion might also be made rigorous. 
the senders at that time. Thus, just because some other structure would have become the signal for $C$ if only senders had started off with a disposition to produce that structure under such-and-such conditions, it does not follow that the structure counts as a genuine alternative. ${ }^{16}$

We are of course aware that this notion of a biologically realistic situation is vague. But we are inclined to see this as a feature rather than a bug of the account; it (partly) explains the diverging intuitions over arbitrariness that we encounter in this area. At the same time, we think there are some necessary conditions that more or less everyone is likely to agree on. In particular, we think that attributing to senders a disposition to perform a certain act (broadly construed) at the time of signal creation is biologically realistic only if that act was within their behavioural repertoire at that time. Thus, a situation in which senders were disposed to perform an act that would have required novel perceptumotor or cognitive capacities, for example, would not count as a biologically realistic situation; a disposition to trace with one's finger in the air the shape of some object would not count as biologically realistic for senders lacking finegrained motor control of their fingers, and so on. In addition, at least in the case of animal signals, ${ }^{17}$ one might accept something like the following necessary condition: at the start of signal creation, at least some members of the population were in fact disposed to produce the alternative structure in response to the same conditions others were disposed to produce $S$. What work might this do? Suppose an act of teeth-baring

${ }^{16}$ When we are dealing with a sender whose signalling behaviour is psychologically mediated, what counts as biologically realistic will encompass (or simply boil down to) the question of what is psychologically realistic for that individual. (In other words, having such-and-such a psychology is just a specific type of biological feature of a sender, and hence certain departures from that psychology may well violate our clause (ii).)

${ }^{17}$ We do not claim that what is biologically realistic must be spelled out in exactly the same way in each case. 
is used as a signal for anger in some group of animals. Then, even if the act of licking was in the behavioural repertoire of the senders at the start of signal creation, it may well be that none of them were disposed to lick as a result of experiencing anger. Thus, a scenario in which they were disposed to behave in this way would be ruled out. ${ }^{18}$ In any case, we regard it as obvious that requiring the counterfactual situation described by the antecedent of (i) in our definition to be a biologically realistic situation will eliminate many of the alternative structures that are intuitively irrelevant. ${ }^{19}$

On our account, the arbitrariness ${ }_{\mathrm{A}}$ of a signal is an objective feature of that signal. (Or at least it is an objective feature, relative to some specification of what counts as a biologically realistic intervention on the system.) The arbitrariness ${ }_{\mathrm{A}}$ of a signal is not a matter of how many genuine alternatives we intuitively think might have been used in the place of that signal, but of how many genuine alternatives might have in fact been used in its place.

Various factors can constrain the arbitrariness ${ }_{\mathrm{A}}$ of a signal. There are, for example, internal constraints. Most obviously, the perceptumotor capacities of the senders and receivers as they existed initially — what they could sense and do at that timedrastically cut down on the range of genuine alternatives. A structure that would not have been reliably detectable by receivers, whatever its other virtues as a signal, would not have worked. The same goes for cognitive capacities. For example, suppose we are dealing with a population whose members have always been devoid of any

\footnotetext{
${ }^{18}$ To illustrate with an extreme example: even if vervet monkeys would have readily learned to associate a picture of a leopard with the presence of a leopard, a scenario in which other vervets were disposed to produce such a picture in the presence of a leopard is patently unrealistic.

${ }^{19}$ In our view, this characterization of what counts as biologically realistic is more than enough to make progress in the present area (something we hope the remainder of this article shows). However, the desire for a more rigorous account is certainly understandable. There are a number of ways in which this might go. Most obviously, one might appeal to possible worlds and some distance metric over such worlds (Stalnaker [1968]; Lewis [1973]).
} 
understanding of others as intentional systems. Then the set of possible signals will be limited to those structures that senders were disposed to perform for noncommunicative reasons. ${ }^{20}$ Still another type of internal constraint would be preference disagreement between the senders and receivers; a conflict of interest may limit the pool of feasible signals to just those that are hard or impossible for senders to 'fake'. ${ }^{21}$

On the external side: features of the environment itself can constrain the $\operatorname{arbitrariness}_{\mathrm{A}}$ of a signal. Here we (minimally) have constraints imposed both by the communication channel and by the probabilistic structure of the environment. To get a sense of the former, suppose we are interested in the number of alternative structures that might have been used in the place of some actual long-range contact call. A structure that would have been perceivable only over short distances, whatever its other virtues as a signal, would never have worked. Nor would a structure that was not reliably detectable given the ambient background noise. And so on. But there are also considerations of how frequently the behaviourally relevant state being signalled about occurred. This will, among other things, bear upon how the costs of various alternative structures affected their feasibility as signals. The flip-side of all this is that the absence of such internal and external constraints can be understood as contributing to arbitrariness $_{\mathrm{A}}$, as facilitating it. $^{22}$

${ }^{20}$ A situation in which senders were disposed to perform acts whose effect on receivers depends on recognition that the sender is attempting to communicate are plausibly seen as ruled out by clause (ii).

${ }^{21}$ The senders may still have multiple means of producing a signal that is appropriately costly, however, in which case there would still be some arbitrariness $\mathrm{A}_{\mathrm{A}}$.

${ }^{22}$ It is worth briefly contrasting this account of arbitrariness with one suggested in passing by Shea ([2011], p. 186) who proposes that we think about the arbitrariness of a signal in terms of the 'costs associated with moving to a different signal'. While attractive on its face (in part due to its simplicity), we think the proposal raises more questions than it answers. Most obviously, when there are multiple structures that might have been used in the place of an actual signal, which structure do we consider and why? If the costs of moving to signal $S_{1}$ are low or non-existent, for example, but the costs of moving to structures $S_{2}$ or $S_{3}$ are high, then how arbitrary is the actual signal? Moreover, it is unclear how comparisons of arbitrariness across signalling systems is supposed to go, especially when there are different numbers of alternatives to consider for the systems being compared. In addition, Shea's 
It will by now be clear that there is a real issue concerning the epistemology of $\operatorname{arbitrariness}_{\mathrm{A}}$, of how we can know how $\operatorname{arbitrary}_{\mathrm{A}}$ a signal is. For knowledge of this will depend on us correctly apprehending what would have happened in some communication system under various counterfactual conditions. This is not trivial, but nor is it a reason for despair. For one thing, we can gain such knowledge via the comparative method: we look at the sorts of signals that similar animals in similar conditions use to communicate about similar things. (This is, for example, the sort of reasoning we employ when we judge that English speakers might have used 'chat' for cat, as do the French.) In addition, we can build and explore formal models and simulations of various sorts (signalling models in particular, but also causal models more generally) to glean such knowledge. By constructing apt causal models of the communication systems we are interested in-models informed by our best biological and (if applicable) psychological features of the senders and receivers - and seeing how they evolve under various parameter values and perturbations, we can plausibly get a fix on what might and might not have worked and why. And there are also more direct empirical means, such as seeing how animals respond to various alternative structures in the laboratory. These tools can be expected to provide at least ball-park estimates $\operatorname{arbitrariness}_{\mathrm{A}}$, and even better judgements of the relative arbitrariness $\mathrm{A}_{\mathrm{A}}$ of two or more

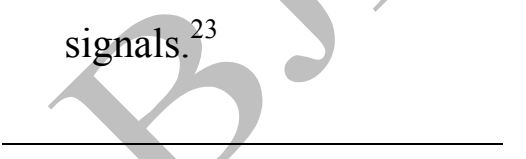

account is silent on the issue of which structures are relevant to our assessment of arbitrariness in the first place. On the present account, costs for the sender or receiver associated with the use of some structure may well influence that structure's status as a feasible alternative to the actual signal. But such costs are just one set of factors among many.

${ }^{23}$ One reviewer suggested to us that often research on the arbitrariness of animal signals seems focused on whether the signal used by some population is 'innate', or the extent to which it is 'innate'. This is indeed an important question to ask in judging the likeness of some form of animal signalling to human language, given that the latter is a paradigm of learned signalling. How does innateness interface with $\operatorname{arbitrariness}_{\mathrm{A}}$ ? The relationship is complicated. Signal $S$ could be significantly arbitrary even $_{\mathrm{A}}$ if the use of $S$ for some content $C$ is innate for the sender. To see this, imagine that at the start of signal creation, there was considerable flexibility in how the sender responded to the relevant stimulus, and 


\section{Arbitrariness ${ }_{R}$}

Often there is quite a different notion of arbitrariness in play in discussions of human and animal communication. This notion is an essentially negative in character: arbitrary signals are signals that are not iconic.

The term 'icon' was introduced by the philosopher and logician Peirce ([1899]). For Peirce, icons were one of three basic types of signals, the other two being indexes and symbols. Peirce conceived of an icon as a signal that referred to its signified (for example, an object or property) in virtue of a perceived resemblance between the signal and its signified on the part of a receiver. He also held that iconic reference was the most basic form of reference in the sense that it was presupposed by indexical reference (which was in turn presupposed by symbolic reference).

The term 'icon' has taken on a looser meaning over time. In particular, theorists often speak of icons without necessarily endorsing Peirce's ideas about the nature of reference and how it is achieved (though this departure from Peirce is often left implicit). This is not surprising, as many of Peirce's ideas in this area have come to look dated in the light of twentieth-century psychology. The most common conception of an icon today is that it is a signal that physically resembles its referent. Among other things, this allows us to talk about icons in the abstract, that is, as separate from a

that the receiver, channel conditions, and so on were all such that more than one of these responses might have gone on to become the signal for $C$. Then, even if the disposition to produce $S$ in response to the stimulus was genetically assimilated over time, it would still be the case that some other structure might have been used in $S$ 's place, and hence $S$ would still count as arbitrary to some significant extent. (Assimilation of this disposition might, however, result in a low degree of forward-looking arbitrariness $\mathrm{A}_{\mathrm{A}}$; see Footnote 15.) On the other hand, if the disposition to produce $S$ in response to the relevant stimulus began as a highly innate feature (if it was strongly developmentally canalized, say), then $S$ would likely have a minimal degree of arbitrariness $_{\mathrm{A}}$. It is also possible for a learned signal to exhibit little or no arbitrariness $\mathrm{A}_{\mathrm{A}}$ (a fictional example is given in Section 5.1), though this is empirically less likely for reasons that will become clearer as we proceed. 
particular interpretative process in the mind of a particular receiver. So, for example, the English word 'bow-wow' is held to be an iconic signal for the barking noise of a dog independently of any particular receiver noting the resemblance between the two.

We see this broadening of the notion of an icon as theoretically useful, but it introduces certain conceptual issues. As was just mentioned, on the Peircean conception, the status of a signal as an icon is determined by its role in interpretation; if it causes the receiver to think of the signified in virtue of resembling the latter, then it is an icon; otherwise, it's not. Thus, two tokens of the same type of signal interpreted by different receivers (or the same receiver at different times) can differ with respect to their status as icons. On the broader conception, in contrast, the status of a signal as an icon is taken to be an antecedent fact, one grounded in physical resemblance between the signal and its content. The physical resemblance between the word 'cough' and the act of coughing, for example, might be noticed by a particular receiver, or it might not. Either way, the word 'cough' is theoretically regarded as an icon (specifically, an onomatopoeia).

There is good reason to reject the idea that physical resemblance between a signal and its content as such, even if it is very salient or specific, is a sufficient condition for that signal to be an icon. Imagine, for example, a population in which the signal for APPLE is red, the signal for BANANA is yellow, and so on. But now imagine that the members of this population are in fact colour blind, and that it is the shape of the signals that does all of the communicative work. ${ }^{24}$ Here there is clear physical resemblance, but it seems wrongheaded to regard the signals as icons. For given the perceptual

\footnotetext{
${ }^{24}$ And, of course, assume that the shapes of the signs do not resemble their referents.
} 
limitations of these agents, they are not even capable of registering the colour of the signals, much less the respect in which the signals resemble contents.

Here is how we see things. There are many ways in which a resemblance between signals and objects or properties elsewhere can play a role in communication. If we take all of these cases to be instances of iconic signalling, then it is doubtful that there is a single, theoretically useful description under which all and only these cases can be brought. Unfortunately, there is not scope to adequately discuss this issue here. What we propose to do instead is simply offer an account that cleanly handles the sorts of cases that those interested in the relationship between animal and human communication tend to focus on. Consider the following:

Icon: Structure $S$ is an icon for content $C$ if and only if: (i) $S$ is a signal for $C$; (ii) $S$ stands in some physical resemblance relation $R$ to $C$; and (iii) that $S$ stands in $R$ to $C$ is manifest to receivers.

The unique part of this account is clause (iii). The notion of 'manifestness' is a technical one we borrow from Sperber and Wilson ([1986]; [2015]). For them, a proposition is manifest to a cognizer at a given time if and only if that cognizer is likely to some positive degree to mentally represent it and accept it as true at that time. Sperber and Wilson talk of 'propositions' rather than 'facts' as they wish to allow that pieces of information that are false to be manifest to cognizers. For present purposes, however, it is only facts that are relevant, and so we will employ that language instead. The critical feature of manifestness is that it does not imply actual mental representation and acceptance. This allows for a resemblance relation between a signal and its content to be manifest to a receiver without him actually making use of this resemblance in 
interpretation or even noticing it. However, that the signal stands in this resemblance relation to its content must be something the receiver is nevertheless capable of mental representing and accepting. Thus, resemblance relations featuring properties that are not perceivable by receivers will not count as manifest to receivers. ${ }^{25}$

Another important feature of the account is this: it does not require that senders intend, nor ever did intend, that receivers recognize that the signal stands in such-andsuch a resemblance relation to its content. If a sender is aware of the fact that receivers make use of resemblances between signals and contents in interpreting signals, then the sender may create a signal that resembles its content for this very reason. But iconic signals can have their origins in far less intelligent processes; they might, for example, start out as (non-communicative) utilitarian behaviours of some kind or 'vacuum behaviours' (Planer [2017]; Sterelny [2017]). For example, imagine a hunter who, upon spotting his prey, imitates the sound of this animal in order to draw it closer. (Suppose that the hunter has observed a conspecific of the prey animal make this noise, and observed that it causes approach behaviour.) This call might alert other individuals in the vicinity to the presence of this animal. If making the prey's presence known to bystanders was advantageous to the sender - say, because it increased the chances of a successful kill - and if the sender recognized that his behaviour tended to have this effect on others, then he might begin to produce this call in the presence of the prey for that very reason (as well). In this way, the sender's call could become a signal for the presence of a certain animal. This is compatible with the sender failing to understand

\footnotetext{
${ }^{25}$ Manifestness can be spelled out in a graded way as well: roughly speaking, a fact is more manifest to a cognizer the more likely he is to mentally represent that fact and accept it as true. We might then say that the iconicity of a signal is proportional to how manifest the relevant resemblance relation is with the signal's content. We think a graded account of iconicity has several nice features, but there is not scope to review those features here. (This is, however, one of the reasons we prefer to formulate the account in terms of manifestness.)
} 
that it is the resemblance between his behaviour and a sound made by the prey that was instrumental in causing others to associate the two.

It is worth contrasting this account with a similar, though considerably stronger account of iconicity. Specifically, one might hold that in addition to being manifest to receivers, mental representation of the resemblance between signal and content on the part of receivers must have actually played a causal role in establishing the signal at some point in time. We regard this as a perfectly sensible suggestion, and have no good reason for opting for our weaker account other than that this allows us to side-step some tricky issues involved in adequately formulating this causal requirement. Other than this, we view the two accounts as equally good ways of answering a question that has hitherto been left open. ${ }^{26}$

This treatment of iconicity is obviously psychologically rich. We are happy with this; it is a way of rendering the original Peircean notion of iconicity—which was itself very psychologically rich-compatible with modern usage in animal and human communication studies. However, one might worry that there are also some common usages of iconicity today that are not tied to receivers' psychology. In particular, two kinds of cases come to mind: threat displays, such as a dog's baring of its teeth, and honeybee waggle dances. We see the former as being covered by our account (at least when those cases involve higher animals) — we do not think it is all that cognitively

${ }^{26}$ This suggestion might bring to mind another for the reader. Specifically, why not simply hold that an iconic signal is a signal that stands in some resemblance relation $R$ to its content $C$, where $R$ either plays, or has played, some causal role in establishing the signal as a signal for $C$ ? This would extend the scope of the notion to cases where agents lack mental representations. We think this is an interesting alternative, but again, some tricky conceptual questions arise in connection with the causal requirement here that we are not altogether sure how to deal with, and that are simply not an issue for the notion we embrace above. Suffice it to say that such an alternative would warrant some different conclusions about the co-occurrence and separability of the two types of arbitrariness we are distinguishing (Section 4), as well as some different conclusions about the cognitive complexity of signal users inferable from the presence or absence of non-iconicity (Section 5). 
demanding for a receiver to recognize that a snarl resembles an actual attack. However, perhaps somewhat contentiously, we would deny that waggle dances are iconic. As onlookers, we can recognize a kind of resemblance between a particular dance and the location it specifies (in the angle of the dance with respect to the vertical of the hive and the angle of the food source with respect to the sun), but it is unlikely that the bees can. And our proposal is that it makes sound theoretical sense to exclude the case on these grounds (it is simply a more complex variant of our colour resemblance case involving colour blind agents above). We do, however, think that waggle dances are likely associated with a low degree of arbitrariness $\mathrm{A}_{\mathrm{A}}$, and hence that the resemblances between dances and the locations they specify are quite robust. It is thus tempting to think of the resemblances in this case as doing some kind of 'communicative work' for the bees. We would suggest that that is the wrong way to read the case. A signal might both resemble its content and be a highly non-arbitrary $\mathrm{A}_{\mathrm{A}}$ signal without that resemblance being recognizable by receivers of the signal. Then, despite this resemblance, the signal would still be non-iconic. Or so we claim. (Some more on this in Section 4.)

We take it that the foregoing discussion gives reasonably precise content to our second notion of arbitrariness. We shall refer to this notion as 'resemblance arbitrariness' and denote it as 'arbitrariness $\mathrm{R}_{\mathrm{R}}$.

Arbitrariness $_{\mathrm{R}}$ : Structure $S$ is an arbitrary signal for content $C$ if and only if (i) $S$ is a signal for $C$; and (ii) $S$ is not an icon for $C$.

Some might find this account of arbitrariness ${ }_{\mathrm{R}}$ too permissive, holding that the notion should be reserved for signals that are not only non-iconic, but also non-indexical. We 
would make just two points in reply. First, while we agree that many theorists - in particular, those who adhere closely to Peirce's original theory-do use the term 'arbitrariness' in this way, many others opt for a notion closer to ours. In fact, a clear example of this can be seen in passage from Price et al. ([2015], pp. 1-2) quoted in the introduction: 'Because the vervet monkey alarm calls bore no resemblance to their respective predator category, in the sense that they did not mimic the sounds made by the respective predators, they were deemed to be non-iconic and thus arbitrary'. This also seems to us to be the more common usage among linguists these days (see, for example, Dingmanse et al. [2015]).

Second, the term 'index' is used in two importantly different ways. On one usage, an index is a signal that is linked to its content by virtue of a 'natural' or 'physical law' (Price et al. ([2015]) use 'index' in this sense). The standard example that is used to illustrate this notion is the connection between smoke (signal) and fire (content). On the other usage, an index is a signal that is produced in the spatiotemporal presence of its content (see, for example, Deacon [1999]; [Rosanno] 2010). These two notions are in fact quite different, and would yield quite different notions of arbitrariness $s_{R}$. When animals develop a signal for a certain predator, for example, that signal is not based on a 'natural law', but rather some contingent process of sender and receiver co-evolution. This contingency is clearest in cases where arbitrariness $\mathrm{A}_{\mathrm{A}}$ is high, but is always present to some degree when we are dealing with co-evolved behaviours. Thus, on the first notion of index, the vast majority of animal signals (and perhaps all of them, depending on how one understands the notion of a 'signal', exactly) will turn out to be nonindexical. Matters are just the opposite on the second notion of an index, however. For the vast majority of animal signals are used in the spatiotemporal presence of their contents (for example, predators, mates, and so on). (The most obvious exception here 
is the waggle dance, where the coincidence of signal and content is limited to the temporal dimension.) Given the lack of consensus on what exactly an index is, and given this wide discrepancy in which signals count as indexes, we find our simpler notion arbitrariness $S_{\mathrm{R}}$ preferable.

\section{A Comparison of Arbitrariness ${ }_{\mathrm{A}}$ and Arbitrariness $_{\mathrm{R}}$}

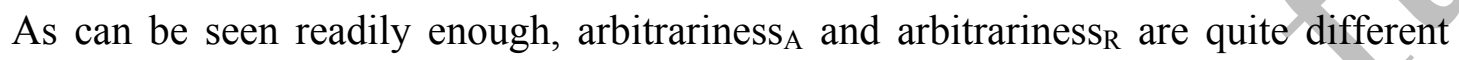
notions. In this section, we briefly consider how they can come apart and coincide.

(i) Arbitrariness $_{A}$ and non-arbitrariness $s_{R}$ : There are many instances of non-arbitrary ${ }_{R}$ signals that exhibit moderate arbitrariness $\mathrm{A}_{\mathrm{A}}$. To give a simple case: imagine two people, $A$ and $B$, who lack a common language, and who are attempting to communicate about a particular tool. Specifically, imagine that $A$ wants $B$ to hand him the saw. As a result, $A$ moves his arm backwards and forwards as if using a saw. $B$, recognizing the resemblance between this motion and the motion one makes while sawing, grasps $A$ 's intention and hands the saw to $A$.

$A$ 's signal in this case is iconic: it stands in a certain physical resemblance relation to the saw (specifically, a movement pattern exhibited by the saw when it is used), and this resemblance relation is manifest to the receiver. At the same time, however, the signal is also plausibly understood as exhibiting a non-trivial amount of arbitrariness ${ }_{\mathrm{A}}$. There are several other acts that had $A$ performed them instead, would have also succeeded in communicating $A$ 's request to $B$. $A$ might, for example, have imitated the sound that a saw makes while cutting, or traced the jagged teeth of the saw with his finger in the air, or pretended that his right hand was a saw, proceeding to 'cut' his left 
hand with it. We take it that all of these would count as biologically realistic things for $A$ to do in such a case, and hence each counts as a genuine alternative to the actual signal.

The same reasoning extends to iconic words. Consider again the English word 'bow-wow'. English contains a number of other icons for the same thing: 'woof', 'ruff', 'bark'. Now, given that each of these words is in actual use in English, the use of one rather than another may have some communicative significance in a particular context. But the point that matters here is this: any of these words, had they been produced in the place of 'bow-wow' at the time the latter was coined, would have worked as signals for the barking noise of a dog, and to imagine such initial conditions is certainly not outlandish. Thus, 'bow-wow' is a clear case of a non-arbitrary $\mathrm{R}_{\mathrm{R}}$ signal possessing a

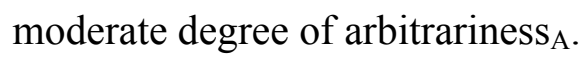

(ii) Non-arbitrariness ${ }_{\mathrm{A}}$ and arbitrariness $\mathrm{R}_{\mathrm{R}}$ : The two types of arbitrariness come apart in the opposite direction as well. This is unsurprising, once the notion of arbitrariness $_{\mathrm{R}}$ is clearly spelled out.

Imagine some species of bacteria that uses a particular protein, $G$, as a signal for a certain environmental toxin, $T$. This 'choice' of $G$ might have been forced by strong bio-chemical constraints. Suppose, for example, that at the start of the process of signal creation, the bacteria possessed just a single type of protein that could simultaneously exit the bacteria's body and survive in the presence of $T$. This protein was $G$. All of the other proteins they possessed at that time either could not exit the body or were dissolved by $T$. Some of the bacteria were disposed to dispel $G$ in the presence of $T$, 
while others were disposed to dispel some other protein that was immediately degraded, or no protein at all.

We are inclined to see this case as one where $G$ is non-arbitrary (that is, has zero $\left.\operatorname{arbitrariness}_{\mathrm{A}}\right)$. Given the total absence of any other protein capable of functioning like $G$ at the start of the process of signal creation, there is a real sense in which, if the bacteria were going to signal about the presence of $T$ at all, they were going to have to use $G$ to do so. The case can be strengthened by assuming that the absence of such a protein was far from coincidental: suppose that given the genes possessed by the bacteria at that time, and even nearby relatives of those genes, it was just not possible for them to synthesize a protein capable of functioning like $G$. Thus, a counterfactual situation in which, at the start of the process of signal creation, senders were disposed to produce some functional analogue of $G$ in the presence of $T$ is plausibly interpreted as violating our requirement that the situation be a biologically realistic one, given the actual properties of the senders at that time.$^{27}$ Of course, one might wish to adopt a more liberal conception of what counts as biologically realistic at this point. But it seems to us that to allow for such a change would be to imagine a fundamentally new behavioural capacity. The problem with this is that once such changes are allowed in, it is unclear where to stop, and the very notion of arbitrariness ${ }_{\mathrm{A}}$ begins to look theoretically empty. ${ }^{28}$ All this having been said, for the purposes of this article, it is not important to us that the case count as one in which there is literally no arbitrariness $\mathrm{A}_{\mathrm{A}}$.

\footnotetext{
${ }^{27}$ We would offer this case as counterexample to the idea mentioned in the introduction that arbitrariness is a necessary condition on signalhood. However, to properly defend its status as a genuine counterexample would take us too far afield at this point. We leave that task for a future work.

${ }^{28}$ Certainly, if one allows, as some authors do (for example, Skyrms [2010]; Cao [2012]; Lepore and Stone [2015]), for significantly different evolutionary histories in advance of the process of signal creation, then every signal will turn out to be highly $\operatorname{arbitrary}_{\mathrm{A}}$, rendering the notion completely empty.
} 
But we do think it should be minimally regarded as an uncontroversial case of very low $\operatorname{arbitrariness}_{\mathrm{A}}$.

In any event, it is obvious that the signal in this case is not an icon and hence counts as arbitrary $_{\mathrm{R}}$. The signal does not stand in any physical resemblance relation to the toxin, but even if it somehow did, it would not matter. This is for the simple reason that such a resemblance relation would not be manifest to the bacteria; they are not capable of mentally representing anything, much less that the signal resembles some object or property in such-and-such a respect. Thus, $G$ is a clear case of an arbitrary $\mathrm{R}_{\mathrm{R}}$ signal that is also non-arbitrary ${ }_{\mathrm{A}}$, or at least minimally arbitrary . $_{\text {. }}$

Moving 'upwards' from this level, many animal signals will also turn out to be $\operatorname{arbitrary}_{\mathrm{R}}$, though many of those same cases will exhibit a non-trivial degree of arbitrariness $\mathrm{A}_{\mathrm{A}}$. The 'chutter' produced by Vervet monkeys in response to snakes is a good example: an arbitrary $_{\mathrm{R}}$ signal that is also arbitrary ${ }_{\mathrm{A}}$ to a significant extent. These cases trend towards our next category.

(iii) Arbitrariness $\mathrm{A}_{\mathrm{A}}$ and arbitrariness $\mathrm{R}_{\mathrm{R}}$ : Human language words are paradigmatically arbitrary and arbitrary ${ }_{\mathrm{R}}$. To take again the word 'dog' as our example: there is a huge range of alternatives that might have been used in the place of this word, and there is no physical resemblance relation between 'dog' and dogs. As was mentioned in the introduction, we suspect that the paradigm status of human language words with respect to both forms of arbitrariness is part of why the two notions of arbitrariness are often run together.

(iv) Non-arbitrariness $\mathrm{A}_{\mathrm{A}}$ and non-arbitrariness $\mathrm{R}_{\mathrm{R}}$ : Real-life cases of signals that fit squarely within this category will be rare. This is because populations that are 
cognitively equipped to mentally represent resemblances between a signal and other objects or properties will tend to also exemplify cognitive and behavioural flexibility of a sort that would have allowed for other structures to be used in the place of the actual signal (more on this in the next section). Hence, in general, where we find non-arbitrariness $\mathrm{R}_{\mathrm{R}}$ we should expect to find at least some degree of arbitrariness $\mathrm{A}_{\mathrm{A}}$.

There is, however, one large set of cases that if one is willing to construe 'signal' and 'communication' broadly enough, could be understood as fitting this mould: demonstration. Suppose that one individual, $A$, wishes to show another individual, $B$, a specific method for processing some food item, say, a coconut. Here the whole point is put on display a particular action sequence. The very nature of the situation thus seems to rule out the possibility of alternative sequences being used in the place of the actual one. Of course, one could recover some degree of arbitrariness $\mathrm{A}_{\mathrm{A}}$ here by adopting a very fine-grained set of criteria for individuating different structures (act sequences), if one wishes. But it might be theoretically illuminating to view these cases in the other way. (See Table 1 for a summary.)

\section{Arbitrariness $_{\mathrm{A}}$, Arbitrariness $\mathrm{R}$, and Cognitive Complexity}

In the remainder of this article we turn to the connections, or lack thereof, between these forms of arbitrariness and cognitive complexity. Here we use the term 'cognitive complexity' in a rough and ready way so as to encompass both the cognitive capacities of signal users and how developed those capacities are. So, for example, an individual, $A$, who possesses all the cognitive capacities of some other individual, $B$, plus, for example, causal reasoning, will count as more cognitive complex. And another 
Table 1. A summary of some of the examples just given, with the graded nature of arbitrariness $\mathrm{A}_{\mathrm{A}}$ suppressed. (The '?' in the bottom-right cell reflects our uncertainty as to whether this should be counted as a genuine instance of the corresponding category.)

\begin{tabular}{|c|c|c|}
\hline & Arbitrariness $_{R}$ & $\begin{array}{l}\text { Non-arbitrariness }{ }_{R} \\
\text { (iconicity) }\end{array}$ \\
\hline Arbitrariness $_{\text {A }}$ & 'dog' & 'bow-wow' \\
\hline Non-arbitrariness $_{A}$ & bacterial signal $G$ & $\begin{array}{c}\text { Demonstration of } \\
\text { coconut-processing } \\
\text { method (?) }\end{array}$ \\
\hline
\end{tabular}

individual, $C$, who possesses a more sophisticated version of causal reasoning would count as more cognitively complex than $B$.

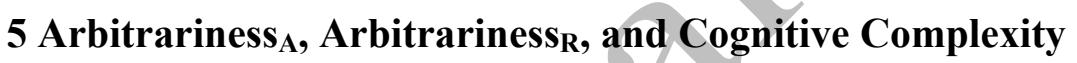

In the remainder of this article we turn to the connections, or lack thereof, between these forms of arbitrariness and cognitive complexity. Here we use the term 'cognitive complexity' in a rough and ready way so as to encompass both the cognitive capacities of signal users and how developed those capacities are. So, for example, an individual, $A$, who possesses all the cognitive capacities of some other individual, $B$, plus, for example, causal reasoning, will count as more cognitive complex. And another individual, $C$, who possesses a more sophisticated version of causal reasoning would count as more cognitively complex than $B$.

\subsection{Arbitrariness A $_{A}$ and cognitive complexity}

How, then, does arbitrariness ${ }_{\mathrm{A}}$ relate to cognitive complexity? Although this is an empirical question, it seems fair to speculate that the two are positively correlated: increasing arbitrariness ${ }_{\mathrm{A}}$ presumably scales with increasing cognitive complexity. This 
basically follows from the fact that with greater cognitive complexity comes greater cognitive and behavioural flexibility. On the sender side, this will tend to mean there is larger range of alternative structures whose production in the place of the actual signal at the start of the process of signal creation will count as biologically realistic. If senders are really intelligent-if, for example, they possess sophisticated 'theory of mind' abilities - then any structure that they could reasonably believe might succeed in communicating their message will count as a biologically realistic structure for them to produce.

Greater cognitive and behavioural flexibility is important on the receiver side too, of course. The former will allow for more sophisticated interpretation of the sender's behaviour: information pertaining to the current context, as well as information acquired elsewhere and elsewhen may be brought to bear. And greater behavioural flexibility will tend to mean that receivers are capable of doing more with the information that their interpretation yields. These factors increase the chances that receivers will be rewarded for paying attention to what senders are doing. If there is also some benefit to senders, conditions are favourable for the emergence of a new signal or set of signals.

It is thus no surprise that humans - the most cognitively and behaviourally

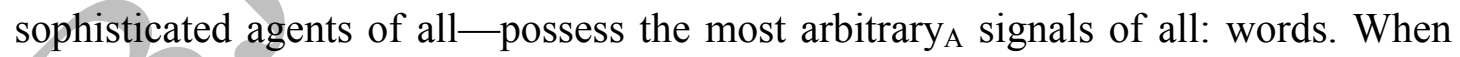
both sender and receiver understand the cognitive processes involved in sign use on the other side, and when both are capable of tracking common ground, there will be a great deal of robustness in the system. Sender and receiver may use 'dog' to mean DOG, but 
if only senders had started out using some other structure, for example, 'cat', ${ }^{29}$ then 'cat' would have become the signal for DOG.

However, we emphasize that this is only a trend, and a rough one at that. It is certainly possible for cognitively simple agents to make use of signals that are arbitrary $_{\mathrm{A}}$ to a significant extent. How so? It may be, for example, that sender and receiver interests are perfectly aligned, in which case there is no need for the signal to be costly (and hence hard to fake). In addition, the channel conditions might also be such that a variety of structures — sounds, sights, perhaps even smells - are all capable of being reliably perceived by receivers. In short, even if cognitive complexity is absent, a range of other conditions favourable to arbitrariness $\mathrm{A}_{\mathrm{A}}$ may be present.

The flip-side to this is that even cognitively complex agents may sometimes be sharply constrained in the range of structures that are usable signals, implying a relatively low degree of arbitrariness ${ }_{\mathrm{A}}$. Imagine, for example, a pair of prisoners who wish to communicate when a guard is approaching, but must do so secretly. They develop a system in which each bangs the water pipe in his cell upon seeing the guard approach. (This works, suppose, because the water pipes sometimes ping on their own. ${ }^{30}$ ) Here channel conditions are maximally unconducive to the use of alternative structures: given the limited range materials available to them in their cells, given the perceptual and cognitive capacities of the guard, and so on, they are more or less forced to use this signal, or else something very much like it.

So, while increasing arbitrariness $_{\mathrm{A}}$ is associated with increasing cognitive complexity, there are possible and real exceptions in both directions. This is not

\footnotetext{
${ }^{29}$ Here we assume that 'cat' was not already in use.

${ }^{30}$ This means that their system will contain false positives, but we suppose the costs and benefits to communication are such that attending to the signal is still beneficial.
} 
surprising, once we recognize that there is a range of factors other than cognitive complexity that can constrain or facilitate the use of alternative structures in the place of the actual signal. Two signals might be comparably (non-)arbitrary ${ }_{\mathrm{A}}$, though for very different reasons. Thus, in assessing claims of human-nonhuman continuity involving $\operatorname{arbitrariness}_{\mathrm{A}}$, attention must be paid to why the cases under discussion are arbitrary $\mathrm{A}$ to the extent that they are, not just how arbitrary $y_{A}$ they are.

\subsection{Arbitrariness $S_{R}$ and cognitive complexity}

What about the relationship between arbitrariness $S_{R}$ and cognitive complexity? The answer is complicated. For starters, as we have already noted, it is perfectly possible for agents devoid of intelligence to make use of arbitrary ${ }_{R}$ signals. Indeed, this is trivially true, given that our definition of iconicity requires that users are capable of detecting resemblances between signals and objects or properties that they might stand for. If some population of agents lacks a psychology altogether, then if they use any signals at all, they will of necessity be arbitrary signals. $^{2}$.

But, of course, agents devoid of psychology will not learn the content of the signals in use in their population; they will come hard-wired to produce and respond to them in particular ways. So, what about cases of learned signals? Are learned, arbitrary ${ }_{R}$ signals a marker of cognitive complexity? There is clearly something to this idea, but matters are more complex. The fact that the signals are $\operatorname{arbitrary}_{R}$ tells us that their users cannot learn the content of the signals by detecting resemblances between signals and contents. But from this it does not follow that the agents must be capable of sophisticated forms of learning or reasoning. If, for example, a signal for a certain type of object is regularly produced in the presence of that object (and not otherwise), and 
receivers are disposed to attend to this object when it is present, then no more than simple association formation is required to learn the content of the signal. ${ }^{31}$

Cognitive complexity picks up where these sorts of cases leave off. When signals are learned, and when they are routinely used to communicate about things elsewhere and elsewhen — when they exhibit so-called displaced reference - then arbitrariness $\mathrm{R}$ is indeed an indicator of cognitive complexity. To learn such signals, users must draw upon information that is not available in the current perceptual environment (which includes the signal and its perceptible features). Exactly what kind of information must be added will differ depending on the details of the learning process and may further depend on the signal that is being learned. But the thread uniting these cases is that no simple process of association formation linking the signal with objects or properties in the present environment will do.

Thus, in assessing claims of human-nonhuman continuity involving arbitrariness $\mathrm{R}_{\mathrm{R}}$, attention must be paid to other features of the signals under discussion. Just because

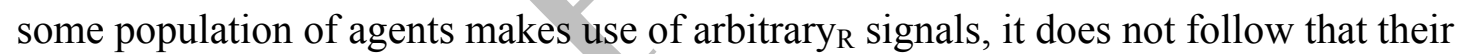
communication system taps cognitive mechanisms similar to those involved in human linguistic communication. But if the signals are learned, and if they exhibit displaced reference, then the claim for continuity is prima facie strong we think.

We take all of this to be more or less straightforward. What complicates matters is that non-arbitrariness $\mathrm{R}_{\mathrm{R}}$ is also a marker of cognitive complexity. Let us explain. Our account of iconicity is compatible with the existence of icons where the resemblance

${ }^{31}$ These are the sorts of cases theorists are attempting to rule out when they take arbitrariness to further imply a lack of indexicality (construed as a signal used in the spatiotemporal presence of its content). The thought is that only signals that are arbitrary in this sense are truly 'symbolic' (see especially Deacon [1998]). For reasons already explained, we prefer our alternative set-up, and would further take issue with this definition of 'symbolic'. But the thing to note is that we can make the same sorts of distinctions and divisions while sticking with our weaker account of arbitrariness $\mathrm{R}_{\mathrm{R}}$. 
between signal and content, despite being manifest to receivers, plays no actual role in the process of signal learning. How might this go? It might be that while there is such a resemblance, the signal is used to draw attention to something that is extremely contextually salient to receivers, or to request a behaviour that receivers are already very likely to perform in the context. Then, the receiver might guess the content of the signal simply on the basis of contextual information together with the fact that the sender is attempting to communicate. The resemblance with the content would be there but unused.

Put another way, then, our account does recognize unexploited iconicity. But to the extent that we find iconic signals remaining stable over long periods of time, the possibility that the resemblance between signal and content is playing no role in the process of signal learning becomes quite implausible. If contextual considerations suffice to make the content of the signal all but obvious to receivers, then the resemblance with the content is likely to fade over time, at least of the original signal was somewhat laborious to perform. ${ }^{32}$ Thus, where we find persistent forms of iconicity, we can infer with some confidence that the receivers somehow make use of this resemblance in the process of interpretation. This is a clear step in the direction of greater cognitive complexity, as it indicates greater cognitive flexibility and sophistication in interpretation.

Above we also said that our account of iconicity allows for icons where the resemblance between signal and content was not intentionally put there by senders for the receiver to notice. The point we now wish to make is that while this is indeed possible, there are also limits to the kind of iconic signals that can be expected to arise

\footnotetext{
${ }^{32}$ Experimental work bears this out (see, for example, Garrod et al. [2006]; Fay et al. [2014]).
} 
in this way. The clearest cases are ones in which the sender is disposed to perform some behaviour that resembles its (eventual) content for (non-communicative) utilitarian reasons. The example we gave earlier had this character; there, we imagined a signal for the presence of a certain prey animal arising out of the sender's antecedent disposition to imitate the sound of the animal in order to call it forth. It is very hard to see how a similar story might be told about the origins of an 'arm-flapping' signal for a bird or of an anthropomorphic engraving as a signal for a person. It is not easy to precisely articulate the difference between these sorts of cases, but the intuitive thought is that there are some iconic signals the production of which critically depend on senders' appreciation of the fact that receivers are sensitive to resemblances between signals and contents and that receivers are likely to make use of such resemblances in interpretation. This is, of course, quite a sophisticated form of social-cognitive understanding. Hence, we suggest that to the extent that we find iconic signals in use in some group for which it is not obvious how they could have arisen in any other way, we can be reasonably confident that there considerable cognitive complexity on the part of senders.

In sum, both arbitrariness $\mathrm{R}_{\mathrm{R}}$ and non-arbitrariness $\mathrm{R}$ bear connections to cognitive complexity, though each relation is complicated and holds only between certain forms

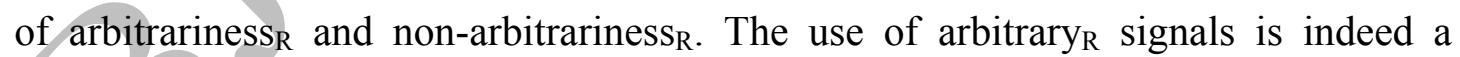
crowning cognitive achievement, but only where those signals are learned and regularly exemplify displaced reference; $\operatorname{arbitrary}_{R}$ signals that do not satisfy these further conditions are compatible with little or no cognitive complexity. At the same time, the use of non-arbitrary $\mathrm{R}_{\mathrm{R}}$ signals is also a marker of cognitive complexity, though only where this iconicity is plausibly regarded as being exploited by receivers. When in 
addition the icons in play resist explanation in terms of non-communicative origins, even more cognitive complexity can be reasonably inferred.

\section{Acknowledgments}

We would like to thank Kim Sterelny, Matt Spike, and the anonymous referees for this journal for their feedback on this manuscript.

Ronald J. Planer School of Philosophy

The Australian National University Canberra, Australia

Ronald.Planer@anu.edu.au

David Kalkman School of Philosophy The Australian National University Canberra, Australia David.Kalkman@anu.edu.au

\section{References}

Artiga, M. [2016]: 'Liberal Representationalism: A Deflationist Defense', Dialectica, 70, pp. 407-30.

Cao, R. [2012]: 'A Teleosemantic Approach to Information in the Brain', Biology and Philosophy, 27, pp. 49-71.

Deacon, T. W [1998]: The Symbolic Species: The Co-evolution of Language and the Brain, New York: W. W. Norton.

Fay, N., Ellison, M. and Garrod, S. [2014] 'Iconicity: From Sign to System in Human Communication and Language', Pragmatics and Cognition, 22, pp. 244-63.

Godfrey-Smith, P. [2000]: 'Information, Arbitrariness, and Selection: Comments on Maynard Smith', Philosophy of Science, 67, pp. 202-7. 
Godfrey-Smith, P. [2013a]: 'Signals, Icons, and Beliefs', in D. Ryder, J. Kingsbury and K. Williford (eds), Millikan and Her Critics, Oxford: Wiley-Blackwell, pp. 4162.

Godfrey-Smith, P. [2013b]: Philosophy of Biology, Princeton, NJ: Princeton University Press.

Godfrey-Smith, P. [2014] 'Signs and Symbolic Behavior', Biological Theory, 9, pp. $78-88$.

Dingemanse, M. et al. [2015]: 'Arbitrariness, Iconicity, and Systematicity in Language', Trends in Cognitive Sciences, 19, pp. 603-15.

Lepore, E. and Stone, M. [2015]: 'David Lewis on Convention', in B. Loewer and J. Schaffner (eds), A Companion to David Lewis, Oxford: Wiley-Blackwell, pp. 31527.

Lewis, D. [2002]: Convention: A Philosophical Study, Oxford: Wiley-Blackwell.

Lewis, D. [1973]: 'Counterfactuals and Comparative Possibility', in W. L. Harper, R. Stalnaker and G. Pearce (eds), IFS: The University of Western Ontario Series in Philosophy of Science, Dordrecht: Springer, pp. 57-85.

Smith, J. M [2000]: 'The Concept of Information in Biology', Philosophy of Science, 67, pp. 177-94.

Owren, M., Rendall, J. D. and Ryan, M. J. [2010]: 'Redefining Animal Signaling: Influence versus Information in Communication', Biology and Philosophy, 25, pp. $755-80$.

Peirce, C. S. (ed.) [1883]: Studies in Logic, Boston: Little Brown.

Planer, R. J [2017]: 'Protolanguage Might Have Evolved before Ostensive Communication', Biological Theory, 12, pp. 72-84.

Price, T. et al. [2015]: 'Vervets Revisited: A Quantitative Analysis of Alarm Call Structure and Context Specificity', Scientific Reports, 5, p. 1322.

Rossano, M. J. [2010]: 'Making Friends, Making Tools, and Making Symbols', Current Anthropology, 51, pp. S89-98.

Scarantino, A. [2013]: ‘Animal Communication as Information-Mediated Influence', in U. E. Stregman, Animal Communication Theory: Information and Influence, Cambridge: Cambridge University Press, pp. 63-88. 
Seyfarth, R. M., Cheney, D. L. and Marler., P. [1980]: 'Monkey Responses to Three

Different Alarm Calls: Evidence of Predator Classification and Semantic Communication', Science, 210, pp. 801-3.

Shea, N. [2011]: 'What's Transmitted? Inherited Information', Biology and Philosophy, 26, pp. 183-9.

Skyrms, B. [1998]: 'Salience and Symmetry-Breaking in the Evolution of Convention', Law and Philosophy, 17, pp. 411-18.

Skyrms, B. [2010] Signals: Evolution, Learning, and Information, Oxford: Oxford University Press.

Sperber, D. and Wilson, D. [1986]: Relevance: Communication and Cognition, Oxford: Basil Blackwell.

Sperber, D. and Wilson, D. [2015]: 'Beyond Speaker's Meaning', Croatian Journal of Philosophy, 15, pp. 117-49.

Stalnaker, R. C. [1968]: ‘A Theory of Conditionals', in W. L. Harper, R. Stalnaker and G. Pearce (eds), IFS: The University of Western Ontario Series in Philosophy of Science, Vol. 15, Dordrecht: Springer, pp. 41-55.

Stegmann, U. E. [2004]: 'The Arbitrariness of the Genetic Code', Biology and Philosophy, 19, pp. 205-22.

Sterelny, K. [2012]: 'A Glass Half-Full: Brian Skyrms's Signals', Economics and Philosophy 28, pp. 73-86.

Sterelny, K. [2017]: 'From Code to Speaker Meaning', Biology and Philosophy, 32, pp. 819-38. 